Nachdruck verboten.

Übersetzungsrecht vorbehalten.

\title{
Zur Kenntnis von Myrmecophilen aus Abessinien.
}

I.

Von

\section{Dr. A. Reichensperger.}

(Aus dem Zoolog. u. vergl.-anatom. Institut der Universität Bonn.)

Mit Tafel 5-6 and 15 Abbildungen im Text.

Im Laufe des verflossenen Jahres (1912) erhielt ich von meinem eifrigen Korrespondenten Herrn Kristensen, Kopenhagen, sehr reichhaltiges Material an Ameisen und Ameisengästen aus Abessinien. Der größte Teil desselben wurde während seines damaligen Aufenthaltes bei der Stadt Harrar und in deren weiterer Umgebung gesammelt. Es ist besonders dankenswert, daß bei der überwiegenden Mehrzahl der Myrmecophilen außer genauen Daten auch stets die Wirte beigegeben waren, deren Bestimmung Herr Dr. SAntschI (Kairouan) in liebenswürdiger Weise kontrollierte und übernahm. Beiden genannten Herren sei auch an dieser Stelle nochmals für ihre Bemühungen Dank gesagt.

Die Ausbeute Kristensen's zeigt wiederum klar, welche vielseitige biologische und systematische Ernte noch des MyrmecophilenForschers harrt. Wenngleich Abessinien wie besonders das benachbarte Eritrea bereits durch RAFFraY's, ANDREINI's und Escherich's Reisen bezüglich der Ameisengäste verhältnismäßig gut durchforscht ist, so befanden sich doch unter dem mir vorliegenden Material eine ganze Anzahl neuer Formen, die im Folgenden beschrieben werden sollen. - Abgesehen von biologisch interessanten gehäusetragenden 
Lepidopteren-Larven, einer unbekannten Ameisengrille u. a. erwiesen sich allein unter den 9 mir zugesandten Arten der Paussiden-Familie nicht weniger denn 6 als neu. Dadurch steigt die Zahl der Angehörigen der Gattung Paussus in engerem Sinne auf 185; aus Abessinien und Eritrea sind nunmehr 27 Paussiden bekannt, von denen 22 sich auf dieses Gebiet beschränken dürften. Leider weist unsere Kenntnis der Paussidenwirte noch sehr große Lücken auf. Dieselbe ist unentbehrlich, wenn wir das Verhältnis der Abhängigkeit von Gast zu Wirt und seine interessanten Folgeerscheinungen näher ergründen wollen. Insbesondere wäre es unter Umständen entwicklungsgeschichtlich von Bedeutung zu erfahren, welche Wirte eine weitverbreitete Paussus-Art an den Gegenpolen ihrer Verbreitung besitzt oder welche Wirte nächstverwandten Paussus-Arten zukommen. Vielleicht ergäbe sich dann eine nähere Erklärung für die fast unbegrenzte Mannigfaltigkeit der Gestalt des Paussidenfühlers sowie eine befriedigende Auskunft über die Rolle, welche den Wirten etwa bei dessen Ausbildung zustand und wohl noch zusteht. Bei weitem die Mehrzahl der bisher bekannt gewordenen Wirte gehört der Myrmicinen-Gattung Pheidole an; auch die abessinischen Arten von Pheidole beherbergen nach Kristensen's Funden vielfach Paussiden, ferner finden sich solche aber nicht selten bei einer kleinen Camponotine, Acantholepis, die auch zahlreiche Gäste aus anderen Gruppen besitzt. -

Herrn P. E. Wasmans bin ich für die photographische Aufnahme der Typen neuer Arten (Taf. 5) sowie für bereitwilligst zur Verfügung gestelltes Vergleichsmaterial seiner Sammlung, Herrn Prof. R. Gestro für den Vergleich zweier eingesandter Arten mit Stücken des Museo Civico in Genua sowie für sonstige Auskunft zu lebhaftem Dank verpflichtet.

\section{Paussidae.}

\section{Arthropterus pallidus RAFFRAY.}

Die Gattung Arthropterus, deren Mitglieder einerseits in Australien (über 50 Arten), andrerseits in Afrika (4 Arten) sich finden, gehört größtenteils dem extremen Trutztypus an. Da sie sich seltener in den Ameisennestern selbst als vielmehr in deren näherer Umgebung aufhalten dürften, ist es nicht erstaunlich, daß bisher nur von 2 australischen Arten bestimmte Angaben über den Wirt vorliegen. 
Von den afrikanischen Arten, die übrigens im allgemeinen Habitus derart von den australischen abweichen, daß die Aufstellung zum wenigsten einer neuen Untergattung berechtigt wäre, fehlten Wirtsangaben ganz. - Meine Exemplare von $A$. pallidus wurden in Nestern von Messor barbarus L., punctatus For. (2 Stück) und von Acantholepis capensis MAYR, canescens Em. gefunden. Dire Daoua und Abuker, März und Mai. - Ob beide, verschiedenen Unterfamilien angehörende Ameisen gesetzmäßige Wirte sind, erscheint mir zweifelhaft, wenngleich Gäste des allgemeinen Trutztyps aus naheliegenden Ursachen weit häufiger mehr- oder vielwirtig sind als Gäste mit Symphilen-Charakter.

\section{Paussus mocemus Gerst.}

Von dieser, gleichfalls dem Trutztypus angehörenden großen Art sammelte Herr Kristensen 2 Exemplare ohne Wirtsameise in der Umgegend von Harrar; Gebel Hakim (2000 m), unter Steinen, Mai.

\section{Paussus kristenseni n. sp.}

(Fig. 4, Taf. 5; Fig. 4, Taf. 6.)

Castaneus, subnitidus, punctatus, piliferus. Antennae validae, articulo primo subquadrato, disperse punctato. Clava oblonga, latitudine duplo longior, lenticularis, lateribus anticis posticisque fere parallelis, perspicue marginatis; supra regulariter perparum convexa, infra in medio inflata, ubique regulariter dense punctata, pilis minutissimis vestita; dente basali brevi valido.

Frons antice truncata, margine paullo elevato, fere plana, parte anteriore polita, posteriore grosse punctata, pilifera. Oculis valde prominentibus. Prothorax irregulariter punctatus, simplex (non bipartitus), antice rotundatus, postice rectus, in medio paullo coartatus et linea transversim impressa, linea longitudinali media brevissima. Elytra elongata, thorace duplo latiora, sicut pygidium dense punctata, punctis breve piliferis. Pedes validi non dilatati aut compressi.

Long. 9,5-10, lat. 3,5. $\mathrm{mm}$.

P. kristenseni, zu der großen Gruppe mit linsenförmiger Fühlerkeule ohne Stirnhorn oder Stirnpore gehörend, nähert sich in der Form der Fühler und allgemeinem Habitus dem $P$. abessinicus Raffr. Er unterscheidet sich deutlich von ihm dadurch, daß auf der Fühlerkeule keine Furchen wahrnehmbar sind, ferner ist der Kopf flacher, nach vorn sehr wenig abfallend, gerade abgeschnitten mit etwas 
aufgebogenem Rande und Ecken. Der vordere Teil des Thorax ist flacher, oben kaum gerundet und ohne vollständigen seichten Längseindruck, vielmehr nur mit einer unmittelbar vor dem Hinterende beginnenden, ganz kurzen, scharf eingerissenen Längslinie, welche die Querfurche schneidet und sich auf dem hinteren Prothoraxteil kurz fortsetzt. Dieser ist fast ebenso breit wie der vordere Teil und nur sehr wenig kürzer. In der Thoraxmitte ist die Punktierung und Behaarung spärlich, diese Stelle ist glänzender als die Umgebung. Flügeldecken parallel, wenig gewölbt, rauh punktiert und am Seitenrande deutlicher behaart. Der Hinterrand der Decken ist stumpfkantig abgestutzt und läßt einen Teil des Pygidiums unbedeckt. - Von dem plumperen $P$. laevifrons Westw. ist die Art durch Form und Skulptur von Fühlerkeule, Thorax u. a. leicht zu unterscheiden.

Abessinien: Harrar. Gebel Hakim (2000 m), Dire Daoua, eine Anzahl von Exemplaren; unter Steinen, ohne Wirtsameise, März-Mai.

Ich widme die Art ihrem Entdecker.

\section{Paussus globiceps n. sp.}

(Fig. 5a, b, Taf. 5; Fig. 5, Taf. 6.)

Fuscocastaneus, subnitidus. Antennae compactae, articulo primo lato globoso, antice paullo marginato; clava vix longior quam lata, antice et apice marginata, postice haut marginata, basi dente brevi valido, paullo recurvato; supra convexa, infra in medio valde inflata; supra nitida, punctis nonnullis piliferis, apice semilunariter opaco, densissime subtiliter punctato. Caput valde globosum, inflatum, antice rectum, fronte concava nitida; vertex valde inflatus densissime pilosus (Textfig. A). Prothorax subinteger, capitis latitudine simplex, antice lateribus rotundatis dense pilosis, postice rectis; in medio transversim impressus, minus pilosus, subnitidus. Elytra arcuata, parallela, thorace vix latiora, dense punctata, pilifera. 우: Pygidium valde prominens, dense punctatum, piliferum; $\widehat{0} \widehat{o}^{\hat{*}}$ : pygidium elytris obtectum. - Pedes perbreves, validi, incrassati.

우: Long. 8,5-9, lat. $3 \mathrm{~mm}$. $\mathrm{o}^{\text {: }}$ long. 5,5 , lat. $2 \mathrm{~mm}$.

$P$. globiceps gehört gleichfalls zu der Gruppe mit linsenförmiger Fühlerkeule ohne Stirnhorn, nimmt in ihr jedoch durch die fast ganz parallele Gestalt und die auffallende Form des Kopfes eine besondere Stellung ein. - Die Fühlerkeule ist kurz gedrungen (Fig. 5, Taf. 6), außen nicht gerandet und ohne merkliche Furchen; 
sie glänzt ziemlich stark mit Ausnahme der Spitze, die halbmondförmig sehr dicht und fein punktiert und dadurch matt ist. - Der Kopf ist hochgewölbt, wie aufgetrieben, oben und seitlich sehr dicht und fein abstehend goldgelb behaart; die Stirn fällt nach vorn steil konkav ab, der abfallende Teil ist hochglänzend, mit geradem, etwas gekieltem Vorderrand und schwach aufgebogenen Ecken. Der Thorax ist breit, der hintere Teil wenig gegen den vorderen abgehoben und verengt, der seinerseits dem Kopf an Breite ungefähr gleichkommt; die obere Fläche ziemlich eben, sehr spärlich zerstreut punktiert, glänzend; die Seiten nebst der vorderen Rundung sind dicht kurz goldgelb behaart.

Die Flügeldecken sind von den Schulterecken seitlich nach unten einwärts gebogen, und ihr Rand ist nach hinten geschweift; überall eingestochen punktiert, mit oben ganz kurzer, seitlich zum Rande zu jedoch länger werdender Behaarung. Das Pygidium tritt beim + vor, ist gleichmäßig punktiert und in der Mitte kurz, seitlich und am Rande etwas länger behaart.

Harrar, Gebel Hakim, März und Juni; mehrere Exemplare, teils ohne Ameisen unter Steinen, teils bei Acantholepis capensis MaYr, canescens Eм. im Nest.

Während $P$. kristenseni gleich den anderen Angehörigen seiner Gruppe einen reinen Trutztypus aufweist, ist bei globiceps ein entschiedener Fortschritt auf den SymphilenCharakter hin gegeben, der sich besonders durch die dichte, wenn auch noch kurze Behaarung zeigt; eine ähnlich stark ausgebildete kommt bei den übrigen bekannten Mitgliedern der Gruppe nicht vor. Auch die parallele gewölbte Gestalt nähert sich derjenigen der höher differenzierten Paussiden. Die

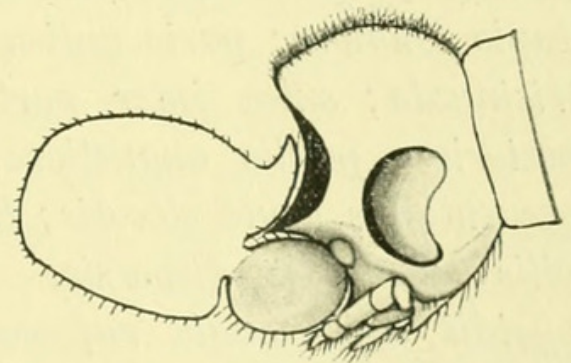

Fig. A. P. globiceps, Kopf von der Seite gesehen. Bildung der Beine deutet noch auf den Trutztypus hin, da ihnen eine weitgehende Verbreiterung eigen ist, im Verhältnis stärker als beispielsweise bei $P$. procerus. Allerdings treffen wir eine solche Verbreiterung ja auch häufig als Schutzanpassung bei den höchststehenden Symphilen der Gruppe mit geteiltem Halsschild.

Bemerkenswert ist bei globiceps der auffallende Größenunterschied der Geschlechter, da im allgemeinen $\delta^{*}$ und $q$ bei den Paussiden kaum verschieden erscheinen. 


\section{Paussus laetus Gerst.}

Diese Art, welche bereits von RAFFray in Höhe von etwa $2000 \mathrm{~m}$ häufig gefunden wurde, ist u. a. von Dr. Andreini ebenfalls mehrfach und zwar bei Acantholepis capensis canescens unter Steinen gesammelt worden. Auch meine zahlreichen Exemplare stammen nur von dieser Wirtsameise her, so daß die Art zweifellos gesetzmäßig bei Acantholepis lebt. In der Färbung der Flügeldecken zeigen sich sehr geringe Variationen, insofern die rötlich-braunen Randpartien bald etwas schmäler, bald breiter sein können. Bei Paussomorphus schwankt nach Gestro (1909) die Ausdehnung der dunklen Flügeldeckenzeichnung viel erheblicher.

Harrar, Gebel Hakim, Bisa Timo (ca. 1300 m). März bis Juni.

\section{Paussus capreolus n. sp.}

(Fig. 3, Taf. 5; Fig. 7, Taf. 6.)

Rufopiceus, elytris nigris; subopacus, thorace nitido. Antennae dense punctatae et granulosae, articulo primo cylindrico; clava perlonga, septies longior articulo primo, paullo recurva, angusta, apicem versus paullo angustata; dente basali brevi, acuto; extus tuberculis sex versus apicem minoribus. - Caput antice paullo depressum, antice bilobatum, vertice utrinque supra oculos foveola parva cicatricosa. Thorace bipartito, capite duplo longiore; parte antica brevi, paullo latiore capite, acute angulata, trisinuata; sulca inter partem anteriorem late aureopenicillata; parte posteriore paullo angustiore, profunde nitidissime impressa; lateribus excavationis acute elevatis; basi recte truncata. Elytra levissime coriacea pilis subalbidis dispersis. Margines laterales elytrorum sicut margo pygidii setis longis rufescentibus ornati. Pedes subnitidi, vix pilosi, solorum posteriorum femora tibiaeque valde compressa et dilatata, pilis subalbidis marginata.

Long. 5, lat. $2 \mathrm{~mm}$.

P. capreolus steht dem P. latreillei Westw. und P. tigrinus Gestro einerseits nahe, andrerseits leitet es von diesen über zu $P$. cerambyx WASM. Mit den ersteren zeigt er einige Übereinstimmung in Färbung und allgemeinem Habitus, unterscheidet sich aber durch die stärker gebogene, viel dünnere und im Verhältnis etwas weniger lange Fühlerkeule von ihnen. Deren Ober- und Unterseite ist wie das 1. Fühlerglied lederartig rauh punktiert, fast geperlt. Die Keule hat etwa ${ }^{5} / 8$ der Gesamtlänge von Kopf nebst Thorax, sie ist messer- 
artig flach zusammengedrückt - viel flacher als bei latreillei und nimmt zur Spitze ganz allmählich an Breite ab. Am Hinterrande nur äußerst geringe Spuren einer Furchenbildung mit 6 unmerklichen Grübchen oben und 6 kleinen Wärzchen unten, die zur Spitze hin an Größe abnehmen und wenige, aber deutliche Börstchen tragen. An der Keulenbasis ein kräftiger Zahn. - Der Kopf ist vorn kurz eingeschnitten und bildet zwei nach innen abgerundete, nach außen mehr stumpfwinkelige, vorn glänzende Lappen. Zum Scheitel sanft ansteigend, sehr dicht fein punktiert. matt. Jederseits befindet sich dicht oberhalb der Augen eine seichte Furche und an deren Hinterende eine schwärzliche kleine Pore. Thorax sehr wenig länger als breit; der vordere Teil ist quer kielförmig, nach hinten stark, nach vorn schwächer abfallend, zerstreut beborstet, mit einer mittleren und jederseits einer seitlichen Furche. Die Basis des Vorderteils reicht weniger weit nach hinten als bei $P$. cerambyx und ist stumpfer. Die sehr breite Querfurche zwischen beiden Thoraxteilen zeigt dichtes, goldgelbes Haartoment. Der abschüssige Teil zur Thoraxgrube hin ist hochglänzend, oben nahe dem Hinterrande sind 2 rundliche Erhebungen. Die Seitenränder der Ausbuchtung des hinteren Thoraxteiles sind schmal erhaben mit gerundet vorgezogenen Vorderecken. - Farbe der Flügeldecken schwarz mit zerstreut regelmäßiger weißer Beborstung. Der Seitenrand trägt sehr starke lange, nach hinten gekrümmte goldgelbe Borsten (fehlen bei $P$. cerambyx). Das Pygidium besitzt lange, dichte Trichombüschel an seinem Hinterrande (fehlen bei P. latreillei). Schenkel und Tibien der Hinterbeine stark verbreitert und zusammengedrückt. Ein näherer Vergleich mit P. tigrinus, Gestro 1901, der, ebenfalls aus Abessinien stammend, von RAFFraY seinerzeit als latreillei (Senegal), bestimmt worden war, ließ sich leider wegen der knappen Diagnose Gestro's nicht durchführen. P. capreolus weicht insofern noch weiter von tigrinus ab, als bei letzterem bereits ,clava antennarum minus incurva, excavatione exteriori paullo latiore" ist als bei latreillei.

Diese Art steht zweifellos auf einer sehr hohen Stufe echten Gastverhältnisses zu ihren Wirten, da außer den Beinen kein Körperteil ohne Exsudatorgan oder Trichome ist.

Harrar, Dire Daoua, März, bei Pheidole rotundata Fon. ilgi Fon. 1 Stïck. 


\section{Paussus anxius n. sp.}

(Fig. 1, Taf. 5; Fig. 6, Taf. 6.)

Fuscopiceus, elytris obscurioribus, opacus, totus breve et disperse albido setosus. Antennarum articulo primo cylindrico, subrotundato; clava lata naviculari; margine antico rotundato paullo obtuse serrato, quattuor foveis impressis; margine postico supra quinque sulcis transversis, obsoletis et obscuris; infra sexcostata, margine costarum penicillato; antennarum clava basi tridentata. Caput thoracis fere latitudine, antice bilobatum, carinatum, hand depressum; in vertice foveis duabus fere triangularibus. Thorax bipartitus, partibus inter se longitudine et latitudine fere aequalibus; parte anteriore lateribus angulatis subdentatis, in medio sulco brevi; parte postica antice profunde excavata et trisulcata. Elytra lata, subquadrata, margine posteriore serie setarum aurearum longarum. Pedes breves, posteriores magis, medii et anteriores minus dilatati et compressi.

\section{Long. 4,7-5 mm, lat. $2 \mathrm{~mm}$.}

$P$. anxius weist sich durch den borstenbesetzten Hinterrand der tiefgefurchten, kahnförmigen Fühlerkeule als zur denticulatus-Gruppe gehörend aus. Im gesamten Habitus sowie in Kopf- und Thoraxbildung vermittelt die Art einen Übergang zu $P$. aureofimbriatus Wasm.; jedoch ist die Gestalt etwas weniger vierschrötig, die Fühlerkeule im Verhältnis um ein Geringes kleiner. Die am oberen wie am unteren Hinterrande ganzrandige Keule ähnelt am meisten der des $P$. excavatus Westw.; sie trägt jedoch unten 6 zur Spitze hin an Größe abnehmende Borstenpinsel; in der Höhlung sind 6 deutliche Furchen vorhanden, 5 sehr schwach angedeutete etwas schwärzliche befinden sich auf der Oberfläche nahe dem Hinterrande. Vorderrand zusammengedrückt, mit 4 deutlichen Grübchen. Die Scheitelbildung ist der von aureofimbriatus ähnlich, aber weniger erhaben, mit 2 deutlichen Poren. Erstes Fühlerglied, Kopf und Thorax sind fein lederartig gerunzelt, punktiert, matt. Der vordere Thoraxteil ist kielförmig mit 3 seichten Furchen, von oben gesehen an den Seiten zahnförmig vorspringend, wenig breiter und kürzer als der Kopf. Seiten des hinteren Thoraxteils kräftig, ziemlich weit nach vorn reichend, so daß die Grube fast rechteckig scharf hervortritt; Trichome nur an deren Rand schwach entwickelt. - Die Flügeldecken sind schwarz, dicht fein punktiert, matt, mit weißen kurzen Borsten belegt; die Decken fallen über das nicht vorstehende Pygidium ziemlich steil nach hinten ab und tragen auf den abfallenden Par- 
tien sowie am Hinterrande spärliche, lange, goldgelbe Borsten, welche zu 3-5 zu einem Pinsel vereinigt sind.

Harrar, Bisa Timo, Mai. 2 mal in Nestern von Pheidole caffra Eм. abyssinica For. je 1 Exemplar.

\section{Paussus modestus n. sp.}

(Fig. 2, Taf. 5; Fig. 8, Taf. 6.)

Castaneus, paullo nitidus, subtiliter punctatus, parvus et angustus. Antennarum articulo primo subquadrato, clava capite vix maior, orbicularis, valde compressa, foliiformis, prope basin paullo incisa, rotundata, dense et subtiliter punctata; postice usque ad tertiam latitudinis partem leviter excavata, margine inferiore valde porrecto, sulcis sex obsoletis transversis, setulis minimis albidis ornato; margine superiore perpaullo cremulato, punctis quinque nigris. - Caput thoracis latitudine, antice paullo carinatum et incisum, vertice supra oculos utrimque breviter cornuto, cornibus inter se carina utrimque incisa connexis. Thorax bipartitus, latitudine paullo longior, lateribus parallelis; pars anterior posteriore paullo brevior; pars anterior lateribus subrotundatis, in medio obsolete impressa, posterior lateribus rectis, angulis anticis obtusis, paullo elevatis breviter aureo-penicillatis; pars anterior magis, posterior minus dense subtiliter punctatae. - Elytra oblonga lateribus impressis, margine laterali praeter basin setis aureis longis reflexis ornato; prope apicem elytrorum serie brevi setarum depressarum. In pygidio series setarum perlongarum albidarum, quarum basis aurea. Pedes valde dilatati et compressi.

Long. vix $4 \mathrm{~mm}$, lat. $1^{1 / 4} \mathrm{~mm}$.

P. modestus, der Gruppe mit blattförmiger Fühlerkeule angehörend, steht dem P. kohli Wasm. einerseits, dem P. bicornis Wasm. andererseits nahe. Von bituberculatus KoLBE unterscheidet er sich ohne weiteres durch die gänzlich plattgedrückte Fühlerkeule, deren seichte Einfurchung außerdem nicht winklig, sondern ganz gerundet vorgezogen ist. In der Art der Furchenbildung ist eine große Ähnlichkeit mit kohli gegeben, jedoch sind keine Basalecken oder Spitzen an der Keule vorhanden, wie bei jenem, vielmehr ist dieselbe überall gerundet, im ganzen fast kreisförmig. Von bicornis endlich unterscheidet sich modestus in der Form der Fühlerkeule, Skulptur des Kopfes, Verhältnis der Thoraxteile zueinander, Art der Behaarung und allgemeiner Färbung.

Auf dem Kopf erhebt sich oberhalb der Augen jederseits ein 
kleines Hörnchen, das mit einer deutlichen länglichen Grube versehen ist. Die Hörnchen sind quer über den Scheitel in doppelter Weise miteinander verbunden: nach vorn zu durch eine stumpfwinklig in der Scheitelmitte gebrochene schwache Leiste; nach hinten durch einen sanft gerundeten glänzenden dunkelbraunen Kiel (Fig. 8 Taf. 6). So entsteht zwischen den Hörnchen ein an der Spitze stumpfwinkliges Dreieck mit etwas konkaver Basis. Der Kopf ist kürzer als breit. Die Thoraxteile sind unter sich ungefähr gleich an Länge wie an Breite, zusammen $1^{3} / 4$ mal so lang wie der Kopf. - Elytren etwas gewölbt, zugerundet, das Pygidium zum Teil freilassend, fein punktiert. Die hinteren ${ }^{2} / 3$ des Seitenrandes sind mit kurzen starken, fast anliegenden goldgelben Borsten versehen, eine halbkreisförmige Reihe ebensolcher Borsten befindet sich jederseits oben auf den Elytren nahe dem Hinterrande. Am Rande des Pygidiums sehr lange stark entwickelte Trichome.

Harrar, Juli. Wirtsameise fehlt.

\section{Hylotorus caroli n. sp.}

(Fig. 6, Taf. 5.)

Ferrugineus, pedibus flavis, parallelus, cylindricus, subopacus. Caput magnum, semiglobosum, dense et regulariter punctatum: frons sulco unico in medio frontis furcato duobus ramis furcae divergentibus, apice cicatricosis. Oculi parvi, anguste reniformes. Antennarum articulo primo perparvo, subquadrato; clava multo majori, ovali, acuminata, apice penicillato, intus prope marginem inferiorem carinata. Frons utrimque ad recipiendas antennas valde excavatus. Thorax punctatus, antice capitis latitudine, postice constrictus; parte anteriori maiori, sulco tenui transverso; parte posteriori antice forte bisinuata, in medio sulco transverso forti, basi recta. - Elytra basi constricta, dorso medio plana, tota levissime coriacea. Pedes breves, femoribus tibiisque maxime dilatatis et compressis.

Long. $5-5,5 \mathrm{~mm}$, lat. $2 \mathrm{~mm}$.

$H$. caroli schließt sich eng an den $H$. blanchardi RAFFr. an, mit dem er im allgemeinen Aussehen und in der Färbung ziemlich übereinstimmt. Er unterscheidet sich von ihm jedoch entschieden durch ganz andere Skulptur, schwächeren Glanz, Form von Kopf und Thorax und Verlauf der Porenrinnen auf dem Vorderkopf. Gleich $P$. blanchardi besitzt caroli zwei Stimporen; während die von diesen auf den Vorderrand des Kopfes zu führenden Rinnen jedoch bei 
blanchardi nur sehr wenig konvergieren und kurz darauf endigen, ohne zusammenzulaufen (RAFFr. 1887, tab. 16, fig. 27), treffen sie bei caroti bald zusammen und gehen in eine gemeinsame schwärzliche, deutliche Rinne über, welche sich bis an den Vorderrand des Clypeus erstreckt. - Der Kopf ist von oben gesehen vorn gerundeter, glänzend, schwach punktiert und zerstreut kurz behaart; seine hintere Hälfte ist dicht grob punktiert, wie der vordere kragenförmige Teil des Prothorax. Quer über diesen verläuft eine breite seichte Furche und von ihrer Mitte aus eine flache Einsenkung zum hinteren Teil des Thorax. - Dieser ist feiner punktiert, mit vorn zwei bogigen Einsenkungen und einer engeren, etwas tieferen rundlaufenden Quereinschnürung hinter der Mitte; seine Basis verläuft geradlinig. Der vordere Thoraxteil ist weder viel länger noch viel breiter als der hintere. Flügeldecken sehr fein lederartig gerunzelt und punktiert, nur scheinbar schwarz gefärbt; in der Tat sind sie heller oder dunkler braungelblich; die schwarze Farbe wird dadurch verursacht, daß der dunkle Körper und die zusammengelegten stark schwärzlichen Flügel durchscheinen. - Die Beine sind sehr stark verbreitert, flach und kurz und können in seichte Ausbuchtungen von Kopf und Körper angelegt werden.

Harrar, Gebel Hakim, Bisa Timo, März bis Juli. Eine Reihe von Stücken aus Nestern der Pheidole megacephala FAB., hin und wieder auch bei Pheidole rotundata MaYr, ilgi For. - Ich widme die Art meinem Vater, der an meinen Bestrebungen stets regsten Anteil nahm, zum 70. Geburtstage.

\section{Die Kopfdrüsen von Hylotorus.}

H. caroli ist die fünfte Art dieser interessanten Gattung, die man wohl als eine parasitische Degeneration des Paussus-Typus ansehen muß. Ihre Mitglieder sind bisher auf Afrika beschränkt: Süd-Afrika 2 Arten, Sierra Leone 1 Art, Abessinien 2 Arten. Der Schutztyp ist aufs höchste entwickelt, da sämtliche Extremitäten nebst den Mundteilen durch Lage und Bau geborgen sind. Mit angezogenen Gliedmaßen ähnelt unser Tier einer kleinen, für Ameisenkiefer unangreifbaren Walze. - Gleichwohl ist der Symphilencharakter noch durch die Stirnporen gekennzeichnet. Die Fühler haben gar keine secretorische Funktion mehr, sind aber ebenso wie die Schenkel und Tibien der Beine überreich mit großen und kleineren Sinnesorganen ausgestattet. Auch der feine Haarpinsel 
an der Fühlerspitze besteht aus Sinnesborsten, welche mit einem mehrfach verzweigten Fühlerlängsnerven in Zusammenhang stehen.

Die Struktur der Stirnporen habe ich auf Frontal- und Tangentialschnitten durch den Kopf näher untersucht, um Klarheit über ihre Funktion zu erhalten. Nach den Befunden ist es gewiß, daß sie der Secretion dienen, und gemäß ihrer kräftigen Entwicklung dürfte ihnen eine bedeutsame Rolle für das Tier zufallen.

Die äußere Porenöffnung ist von einer verdickten Chitinschicht wallförmig umgeben, etwas verengt und von länglich-ovaler Form. Sie bildet den Ausgang einer tiefen becherförmigen Einsenkung; dieselbe verbreitert sich zunächst etwas, wird dann schmäler und geht, wieder breiter werdend, in ein Bodenstück über. Die Wände des Bechers werden bis unmittelbar hinter der Verengung von soliden Chitinlamellen gebildet; dann aber wird das Chitin etwas dünner und ist von zahlreichen größeren und kleineren, selten regelmäßig stehenden Poren durchbrochen. Dieselben liegen meist ziemlich dicht beieinander und sind schwach wallförmig umrandet. Auf diese Art kommt ein umfangreiches Sieb, ein Cribellum, zustande. In dichten Lagen befinden sich rings um die Außenwände des Bechers große Drüsenzellen, von welchen jede einzelne einen sich langsamer oder schneller verengenden Ausführgang zu dem Cribellum hinsendet, wo er sich an eine Pore anschließt. Der Eindruck eines Pseudacinus tritt bei Hylotorus viel weniger deutlich zutage, als das bei den Stirndrüsen von Paussus cucullatus nach Wasmann 1903 der Fall ist.

Die Drüsenzellen sind von oval-birnförmiger Gestalt; ihr Plasmainhalt ist feinkörnig, wenig vacuolisiert; die fast stets rundlichen

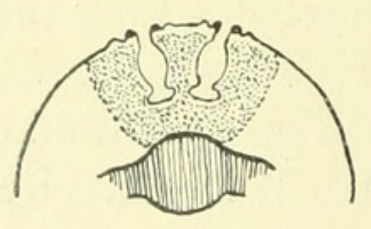

Fig. B. Etwas schräger Schnitt durch den Kopf von Hylotorus. Schraffiert:Gehirn; punktiert: Komplex der Drüsenzellen um die becherförmigenEinsenkungen. Kerne sind mittelgroß und liegen dem inneren Ende der Zellen genähert; sie zeigen mehrere deutliche Kernkörperchen. In den meisten Zellen ließen sich trotz einfacher Alkoholkonservierung des Materials die Drüsenbläschen sehr gut wahrnehmen; sie sind im allgemeinen länglich gestreckt, mit deutlichem Kanal in der Mitte, wie er an dem Querschnitt Fig. 3, Taf. 6 zutage tritt. - Die Länge der Zellen nebst Ausführgang kann bis $0,26 \mathrm{~mm}$ betragen, bei einer größten Breite von $0,04 \mathrm{~mm}$. Nach außen zu wird der ganze umfangreiche Drüsenkomplex von einer einfachen bis doppelten Lage der chitinbildenden Epithelzellen begrenzt, 
welche am Cribellum sehr reduziert erscheinen; nach innen reichen die Drüsen bis an das Gehirn, um das sie sich vorn und oben schwach kapselförmig gewölbt legen. Die gesamte Tiefe der becherförmigen Gruben beträgt etwa bis $0,32 \mathrm{~mm}$, ihre größte Breite $0,11 \mathrm{~mm}$. Sie sind, wie oben erwähnt, paarig vorhanden und konvergieren etwas in ihrem Verlauf nach innen zu. Auf den Schnitten zeigten sich meist im Lumen der Becher noch Secretansammlungen in ziemlicher Menge von fadiger, feinkörniger Struktur (Fig. 5 Taf. 6).

Es unterliegt keinem Zweifel, daß wir in den Elementen des Drüsenkomplexes modifizierte einzellige Hautdrüsen vor uns haben, welche ins Innere des Kopfes verlagert sind. Andere vereinzelte Drüsen, wie sie bei $P$. cucullatus vorkommen, waren im Kopf von Hylotorus nicht zu finden. Auch fehlt hier ein bei jenem vorhandener Kranz von Sinnesborsten um den Porenausgang; nur ganz zerstreute Sinneshaare wurden angetroffen. Hieraus scheint mir ebenfalls hervorzugehen, daß der Trutzcharakter den Symphilencharakter zurückdrängt, wenngleich die Entwicklung der Stimporendrüsen selbst keinerlei regressive Merkmale erkennen läßt. Leider ist noch unbekannt, wie sich die Wirte dem Hylotorus gegenüber verhalten und ob dessen Nahrung im wesentlichen aus Ameisenbrut besteht, was ich nach Untersuchung eines Mageninhalts faßt vermuten möchte.

\section{Clavigeridae.}

\section{Clavigerodes abessinicus RAFFR. (1877).}

In mehreren Stücken aus Nestern von Acantholepis capensis Mayr, canescens Em. Bisa Timo bei Harrar, April und Mai. Von Escherich 1906 bei Acantholepis simplex For. gesammelt.

\section{Clavigerodes raffrayi WASM. (1912).}

In einem Exemplar mit den vorigen zusammen bei der gleichen Wirtsameise. Bisa Timo, Mai.

\section{Articerodes syriacus (SAULC. 1865).}

Mehrere Stücke dieses sehr kleinen Clavigeriden stammen ebenfalls aus einem Nest von Acantholepis cap. can. Harrar, Juni. Die Art hat einen verhältnismäßig weiten Verbreitungsbezirk: Syrien, Mesopotamien, Bokhara, Karatak und Abessinien, wo sie bereits von 
Raffray aufgefunden wurde. SAULCY gibt sie (1874) für Syrien an als: „Parasite d'une petite fourmi, qui vit sous les pierres"; wahrscheinlich handelte es sich um eine Lasius-Art. Dagegen dürften RAFFRAY's abessinische Exemplare bei Acantholepis gefunden sein, wenngleich ihre Etikette den Vermerk "Lasius" trägt (Wasmans, Krit. Verz., 1894), da Acantholepis cap. und Lasius niger gewisse Ähnlichkeit besitzen und erstere in einem Teil von Abessinien in etwa die Stelle zu vertreten scheint, die bei uns $L$. niger einnimmt.

\section{Cossyphodidae.}

\section{Cossyphodes raffrayi (GESTRo 1874).}

Die zahlreichen Exemplare dieses durch die Größe der Augen und durch die Deutlichkeit der Flügeldeckenrippen ausgezeichneten Cossyphodes stammen alle aus Nestern von Acantholepis capensis canescens. Es scheint demnach, daß die Art an diesen einen Wirt gebunden ist, sehr im Gegensatz zu der folgenden. Gestro gibt in seiner Diagnose an: "prothorace in medio haud carinato". Manche meiner Stücke zeigen ganz schwache Spuren einer Kielung vorn und hinten am Prothorax, stimmen im übrigen jedoch vollkommen mit Gestro's Beschreibung überein. Ein Wirt wird bei Gestro nicht genannt.

Bisa Timo, Dire Daoua, März bis Juni.

$1,9-2,5 \mathrm{~mm}$.

\section{Cossyphodinus beccarii (GESTRo).}

Diese Art wurde von Gestro als Cossyphodes beccarii beschrieben, ist aber nach Bildung der Fühler und Mundteile fraglos ein echter Cossyphodinus (WAsм., No. 99). Das Tier repräsentiert den reinsten Schutz- und Trutztyp und dürfte für weitaus die Mehrzahl der Ameisen-Arten absolut unangreifbar sein. Es ist daher wenig erstaunlich, daß es mir mit mehreren Wirten zuging, die sogar zu verschiedenen Unterfamilien gehören.

Harrar und Dire Daoua, März bis Juni. Bei Camponotus maculatus FABR. i. sp. mehrfach; bei Acantholepis cap. can. in einigen Stücken; bei Monomorium afrum Andr. einmal; bei Messor barbarus punctatus am häufigsten.

Die Größe der Art schwankt bedeutend, eine Eigentümlichkeit, die sie mit der indischen $C$. indicus W Asm. teilt: $2.5-3,7 \mathrm{~mm}$. 


\section{Nitidulidae.}

\section{Nitidopecten $\boldsymbol{n}$. g. Nitidulinorum.}

Sulci antennarii recti, paullo convergentes, lati. Labrum profunde incisum. Mandibulae apice bifido. Palpi labiales filiformes. Tarsi anteriores maxime, medii mediocriter, posteriores haud dilatati. Tibiae anteriores margine exteriore subtiliter serrata. Thoracis basis haud marginata, basin elytrorum scutellique obtegens. Prosternum in medio carinatum. Elytra abdomen pygidio excepto obtegentia.

Nitidopecten besitzt viele Ähnlichkeit mit der Gattung Nitidula, vornehmlich in der allgemeinen Körperform und Behaarung, in der Bildung des Kopfes, der Mundteile und der Fühler. Wie bei dieser ist das 1. Fühlerglied nach außen verdickt, und das letzte Glied der rundlichen dreigliedrigen Keule zeigt etwa in der Mitte eine plötzliche deutliche Verengung. - Ganz abweichend ist jedoch die Bildung der Oberlippe, welche tief und ziemlich eng eingeschnitten etwa an die von Epuraea erinnert. Ferner ist das Prosternum in der Mitte rundlich längsgekielt und setzt sich zwischen den Coxen der Vorderbeine in einen sehr kurzen, wenig verbreiterten, gerade abgeschnittenen flachen Fortsatz fort, der bis vorn auf das Mesosternum reicht. Sämtliche Tibien sind etwas von oben nach unten zusammengepreßt, zum Ende hin verbreitert; die vorderen sind zweikantig, die mittleren und hinteren dreikantig, mit zwei nach außen gerichteten Kanten. Der Außenrand der Vordertibien ist sehr fein regelmäßig gezähnelt; die Außenkanten der Mittel- und Hintertibien sind stark beborstet, der Schlußrand ist mit zahlreichen kleineren Dornen und nach innen mit einem langen Enddorn bewehrt. Der Thorax ist am hinteren Ende ganz ungerandet und überdeckt die Basis der Elytren und des Schildchens, das nur schmal, stumpfwinklig zugerundet sichtbar wird. Das Pygidium wird von den Flügeldecken nicht überdeckt. Der Rand des Thorax und der Elytren ist eng und lang beborstet.

\section{Nitidopecten comes n. sp.}

(Fig. 7, Taf. 5.)

Totus piceus, opacus, pedibus antennisque luteobrunneis. Corpus oblongum subdepressum. Labrum profunde incisum utrimque penicillatum. Caput magnum, grosse punctatum, oculis prominentibus. Thorax transversus, lateribus rotundatus, vix marginatus, angulis anterioribus et posterioribus obtuse rotundatis. Thorax elytraque grosse punctata, 
pubescentia aurea vestita, lateribus dense aureosetosa. Elytra anguste marginata, postice rotundata. Abdominis segmenta ultima margine perlonge aureosetosa. Apex tibiarum anteriorum inarmatus, mediarum posteriorumque spinis minoribus 16-20 et spina singula forti armatus (Textfig. C). Tarsorum anteriorum articuli 1-3 valde dilatati, dense pilosi, breves, articulus 4 minimus.

오: Long. 6,5, lat. 3,2 mm. 0: Long. 5,2, lat. 2,6 mm.

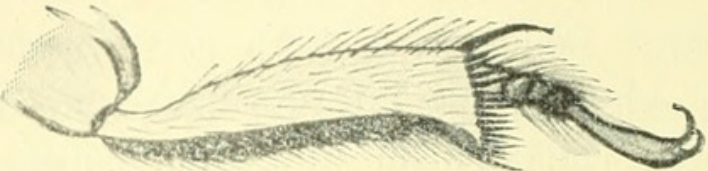

Fig. C.

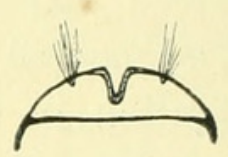

Fig. D.

Linkes Hinterbein von Nitidopecten comes.

Labrum von $N$. comes.

Die auffällige, goldrote, ziemlich lange Pubescenz, welche nicht nur auf der ganzen Oberseite vorhanden ist, sondern auch unterseits sowie an den Extremitäten bald kürzer, bald länger sich vorfindet, sowie die überaus starke, gleichfalls goldrote Beborstung der Ränder von Thorax, Elytren, Pygidium und der Außenkanten der Mittelund Hintertibien geben der Art ein besonderes Gepräge. Es scheint mir keinem Zweifel zu unterliegen, daß die Behaarung im Dienste der Symphilie steht, zumal sich an einzelnen Stellen Randtrichome mit genau ebensolchen Verletzungen vorfanden, wie sie an den Trichombüscheln der Lomechusen und Atemeles durch allzu stürmisches Belecken und Zerren seitens der Wirtsameisen verursacht werden. Die Ähnlichkeit der Rand- und Pygidialtrichome mit denjenigen mancher Paussiden ist geradezu verblüffend. - Der Aufenthalt und die Lebensweise einer ganzen Anzahl von Nitidulinen bringt diese in vielfache Berührung mit Ameisen, und die bei uns heimische Amphotis marginata ist als gesetzmäßig myrmecophil zu betrachten. Bei ihr ist die Behaarung des Körpers jedoch recht spärlich, und sie steht auch durch Fühlerbildung, Ausbildung der Flügeldecken, welche das gesamte Abdomen bedecken, u. a.m. unserer Gattung ferner. Dagegen weisen die meisten Vertreter der Gattung Nitidula außer sonstigen oben erwähnten Übereinstimmungen mit Niditopecten eine Beborstung auf, welche, wenn auch im allgemeinen weit schwächer ausgebildet, doch in den Grundzügen derjenigen unseres Tieres entspricht. Wir können uns letzteres unschwer als myrmecophile Anpassungsform aus ersterer hervorgegangen denken, zumal die Nitudulinen zumeist bereits in ihrer Gestalt und in den 
Deckungseinrichtungen für Fühler und Beine einen allgemeinen Schutztypus aufweisen, der ihnen den Ameisen gegenüber nur vorteilhaft sein kann.

Abessinien, Dire Daoua, März. Beide Geschlechter im Nest von Acantholepis capensis canescens.

\section{Myrmecophile Gehäuseraupen.}

Es ist bereits seit längerem bekannt, daß eine ziemliche Anzahl von Raupeh, meist den Lycaeniden und Tineiden zugehörig, mit Ameisen oder Termiten in ein näheres Lebensverhältnis treten können. Jedoch handelte es sich bisher, soweit Beobachtungen oder Schlüsse ein Urteil zulassen, stets um Raupen, die durch Abgabe von Secreten sich ihren Wirten angenehm erweisen, die also bestimmte Exsudatorgane besitzen und auf Grund dessen von Ameisen oder Termiten außerhalb der Bauten aufgesucht, beleckt und geschützt oder gar in den Bauten selbst gehalten werden. Solche Raupen stehen also mit ihren Wirten im Verhältnis der Symphilie oder der Trophobiose; sie bedürfen beim Verkehr mit den Wirten keines besonderen Schutzes für ihren Körper, da diese ihnen freundlich gesinnt etwa als Leibwache dienen. - Im Laufe des vergangenen Jahres erhielt ich nun einerseits aus Abessinien von G. Kristensen, andererseits aus Süd-Afrika von Dr. Brauns gesammelte, höchst eigenartige Gehäuse aus verschiedenen Baustoffen und von verschiedener Form, welche alle in Ameisennestern gefunden worden waren. Beim Öffnen der Gehäuse stellte sich heraus, daß die Insassen Larven waren, und zwar, wie sich bei näherer Untersuchung ergab, Schmetterlingsraupen. Daß diese Larven weder Symphilen noch Trophobionten sein konnten, ergab sich aus dem Vorhandensein eines festen Gehäuses von selbst; dieselben zeigten sich nach Baustoff und Form wohl geeignet, ihren Insassen vollen Schutz gegen die Ameisen zu gewähren. Es blieben nur zwei Möglichkeiten für das Verhältnis von Raupe zu Wirt offen: dasselbe war entweder ein direkt feindliches oder ein mehr indifferentes. Aus dem Folgenden wird sich ergeben, daß wir eine Raupenart als Synechtren im wahrsten Sinne bezeichnen dürfen, während für die anderen sich das Verhältnis nicht so einwandfrei feststellen läßt.

a) Die Gehäuse.

Sowohl aus Abessinien wie aus Südafrika (Pt. Elizabeth) lagen je zwei ganz verschiedene Arten von Gehäusen vor. Die einen 
waren kleiner, aus einer äußeren Lage ganz regelmäßig kreisförmiger schwarzer oder mehr bräunlicher Plättchen bestehend, welche durch eine innere Schicht feiner Gespinstfäden zusammengehalten wurden. Bei dieser Art zeigte sich in der Form und Bauweise zwischen den abessinischen und südafrikanischen Stücken kaum ein Unterschied, und wir können sie gemeinsam beschreiben; letztere waren im allgemeinen etwas kleiner.

Die Form ist die eines mehr oder weniger gestreckten Rechtecks mit starker Abrundung der kurzen Seiten (Fig. 9, Taf. 5 und Fig. 11, Taf. 6). Ober- und Unterfläche sind ganz gleich, nach der Mitte zu schwach gewölbt, nach dem Rande zu platter, so daß das ganze Gehäuse fast flach und sehr dünn ist. Die Plättchen liegen in geordneten Reihen, sich gegenseitig regelmäßig schuppentörmig deckend. In der Mitte von Ober- und Unterfläche sehr klein, meist heller gefärbt, nehmen sie nach dem Rande zu stetig an Größe zu und sind an den abgerundeten Seiten bei weiten am umfangreichsten. Hier befindet sich vorne und hinten je eine Öffnung, welche die ganze Rundung einnimmt, normalerweise aber hermetisch verschlossen ist. Durch die Führung der inneren Gespinstfäden, welche teils parallel der Rundung, teils schärfer angezogen von den höchsten Punkten der Rundung ins Innere und zu den Langseiten verlaufen, wird nämlich eine Federung bewirkt, welche die sehr dünnen Ränder der Oberfläche und Unterfläche gegeneinander gepreßt hält. Schneidet man eine Langseite auf, so rollen sich die gerundeten Ränder ein. - Die eingehendere Untersuchung der Deckplättchen ergab die merkwürdige Tatsache, da $B$ dieselben aus Chitin bestehen und daß die Raupe das Baumaterial vom Körper ihrer Wirte nimmt! Vorzugsweise entstammt das Material den Hinterleibsringen der Ameisen, und die Plättchen werden mittels der Kiefer ausgeschnitten und durch Gespinst befestigt. Zuweilen erkennt man an denselben die Stelle, an welcher der Ausschnitt begonnen wurde, als etwas weniger regulär gerundet oder als minimalen Vorsprung. Die Struktur des Chitins, die Verteilung der Poren und Börstchen, die man in gleicher Anordnung und Ausbildung wie am Hinterleib der Ameise noch an den Gehäuseplatten mikroskopisch sichtbar machen kann, beseitigen jeden Zweifel über die Identität des Materials. - Daß die Hauptnahrung - wahrscheinlich gar die einzige - der Raupen aus Ameisen besteht, kann uns nach dem Gesagten nicht wundern. Der in stark verdünnter Kalilauge behandelte Raupenkörper zeigt den Darmtractus von Anfang bis zu 
Ende vollgepfropft mit Chitinteilen von Ameisen: ganze Mandibeln und vollständige Fühlergeißeln, welche ihre Zugehörigkeit zur Wirtsameise noch erkennen lassen, Beinstücke, Fragmente von Thorax und Abdomen werden sichtbar. - Die Wirtsameise ist in Abessinien fast stets Acantholepis capensis canescens; nur in einem Falle war Pheidole megacephala als Wirt mitgesandt. Umgekehrt kommt für Süd-Afrika in erster Linie Pheidole capensis MaYR in Betracht, während die Gehäuse dort nur vereinzelt bei Acantholepis capensis i. $s p$. Mayr gefunden werden (briefl. Mitteilung Nr. 17 von Dr. Brauns an Wasmanx 25./4. 1898). Die Färbung der Gehäuse läßt meist ohne weiteres die Bestimmung der Wirtsameise zu; die bei den schwärzlichen Acantholepis gefundenen sind schwarz, abgesehen von einer in der Regel vorhandenen helleren Mittelpartie; die bei den braunen Pheidole vorkommenden sind entsprechend heller oder dunkler braun. Die helle Mittelpartie, welche die kleinsten Plättchen umfaßt, ist der zuerst gebaute Teil des Gehäuses. Die jungen Räupchen werden noch nicht imstande sein, das zähe bereits festgewordene Ameisenchitin zu bearbeiten, und benutzen daher Teile von Puppen oder von noch nicht ausgefärbten weichen Tieren zu ihrem Bau.

Das kleinste abessinische Gehäuse maß 4,8 mm in der Länge zu 1,6 mm Breite; das größte hatte $10 \mathrm{~mm} \times 3 \mathrm{~mm}$ bei $1,2 \mathrm{~mm}$ Dicke; die zu letzterem Gehäuse gehörige Raupe war etwa $7 \mathrm{~mm}$ lang. Die süd-afrikanischen Gehäuse maßen von $5,4 \times 1,5$ bis $7 \times 2,2 \mathrm{~mm}$.

$\mathrm{Ob}$ diese myrmecophagen Raupen sich an gesunde Ameisen wagen, läßt sich schwer beurteilen; jedoch halte ich es für möglich. Dafür spricht der unverletzte Zustand ganzer Fühlergeißeln, die bald nach dem Tode der Ameisen spröde und brüchig werden, im Vorderdarm der Raupen. Vorzugsweise werden sich dieselben allerdings von Puppen und Brut sowie von kranken Ameisen nähren; im Abfallnest sind sie, wie die folgenden, nach Dr. Brauns' und Kristensen's Mitteilungen, gleichfalls zu finden. Soweit sich aus der Untersuchung der Chitingehäuse und ihrer Insassen sagen läßt - die süd-afrikanischen Stücke waren trocken präpariert und daher die Untersuchung der Raupen sehr erschwert - dürfte es sich, trotz der weiten Entfernung zwischen den Fundorten, um dieselbe Raupen-Art handeln. Sollte sich später eine Verschiedenheit ergeben, so wäre den markanten Beispielen für Konvergenz-Erscheinungen, wie sie die Myrmecophilen häufig bieten, ein weiteres hinzuzufügen. 
Aus Port Elizabeth lagen mir von Dr. Brauns ferner drei Sandgehäuse von erheblicher Größe vor, welche Raupen bzw. eine Puppe bargen. Ihre Gestalt erinnert an die eben beschriebenen, ist aber im ganzen mehr elliptisch und unregelmäßiger, nach einer Seite etwas schmaler. Der Baustoff besteht aus Quarzkörnchen, die längeren Seiten sind geschlossen, vorn und hinten wird die Rundung wieder von je einer breiten Spaltöffnung eingenommen, welche in gleicher Art selbstschließend ist. Das kleinste Gehäuse maß $2,4 \mathrm{~cm} \times 1 \mathrm{~cm}$, das größte (Puppe) $3,1 \times 1,2 \mathrm{~cm}$ bei $3 \mathrm{~mm}$ Dicke. Die leere Puppenhülle aus letzterem hatte $1,5 \mathrm{~cm}$ Länge. Die Wirtsameise ist Pheidole capensis MAYR. ${ }^{1}$ )

Endlich gingen mir in großer Zahl eigenartige Sandgehäuse aus Abessinien zu, deren kleinstes $0,5 \times 0,3 \mathrm{~cm}$, deren größtes $1,7 \times 0,9 \mathrm{~cm}$ maß. Die Form derselben ist besonders in jüngeren Stadien breitgerundet oval; später nehmen sie meist an Länge mehr zu als an Breite. Dagegen bleibt ihre Dicke fast bis zur Verpuppung der bewohnenden Raupen annähernd gleich gering, nämlich nur ${ }^{1} / 2-3 / 4 \mathrm{~mm}$. Dieses ganz flache Gehäuse (Fig. 8, Taf. 5; Fig. 9, Taf. 6) wird aus zwei gleichen dünnen Teilen gebildet, welche nur in der Mitte der etwas längeren Seiten an gegenüberliegenden Stellen eingeschnitten und nur hier miteinander fest verbunden, im übrigen aber ganz frei sind. Gleichwohl liegen die freien Ränder der Blätter durch bestimmte Anordnung der inneren Gespinstfäden, die sich zum großen Teil in Bögen zu den Einschnitten hinziehen, einander so fest federnd an, daß es schwer ist, mit einem feinen Skalpell das obere Blatt vom unteren zu heben. Läßt man los, so tritt selbsttätiges Schließen ein. Für die recht flache Raupe hat die Behausung große Vorteile, vor allem ist die schwerere Beweglichkeit, die den meisten Gehäuseträgern anhaftet, vermieden. Überall am Rande, nach vorn wie nach hinten, kann der Kopf vorgestreckt werden, da das Tier sich innerhalb des Gehäuses vollständig umdrehen kann. Die Oberfläche der Wohnung besteht aus feinsten Erd- und Sandpartikelchen, die der Umgebung entnommen sind, das Gehäuse also unscheinbar machen. Dazu ist das Ganze sehr leicht und kann ziemlich rasch fortgezogen werden, wovon ich mich ebenso überzeugte wie von der großen Lebenszähigkeit der Tiere. - Herr G. Kristensen sandte mir auf Bitte gegen Mitte

1) Nach einer mir während des Druckes dieser Arbeit zugegangenen Mitteilung von Dr. BRAUNs kommen diese südafrikanischen QuarzgehäuseRaupen häufiger in Abfallhaufen fern von Ameisen vor. 
Juni 1912 einige 20 lebende Exemplare von Harrar und zwar in einer Streichholzschachtel; 18 überstanden den 3wöchigen Transport gut, und diese hoffte ich teils zur Verpuppung und zum Ausschlüpfen zu bringen. Da mir die eigentliche Wirtsameise, Acantholepis capensis canescens, unzugänglich war, brachte ich die Tiere zu Lasius niger, Lasius flavus und Myrmica laevinodis in LubBock- und JANET-Nester, hielt auch mehrere isoliert im Gipsnest. Letzteren gelang es zu entweichen, obwohl das Nest keine Lücke aufwies, welche einer Myrmica oder einem Lasius-Arbeiter gestattet hätte zu entkommen. Einen der Flüchtlinge fand ich am 16. August im Innern eines lange nicht geöffneten, dicht schließenden Bücherschrankes an einem Buchrücken aufwärts kletternd wieder, ein Beweis, wie vorzüglich die dünnen geschmeidigen Gehäuse sich zur Bewegung durch sehr enge Spalten und Gänge eignen. - Die kleinen Kolonien von $L$. flavus und Myrmica besaßen keine Brut; die eingesetzten Raupen wurden gänzlich ignoriert und bewegten sich noch mehrere Wochen herum, dann gingen sie vielleicht aus Nahrungsmangel zugrunde. Die Bewegungen gehen ziemlich rasch vor sich; sichtbar werden außer dem Kopf meist 2 Brustringe und die Vorderhälfte des 3. Bei plötzlicher Berührung oder Belichtung erfolgt ein blitzschnelles Zurückziehen, ähnlich wie bei unseren Clytra-Larven. Von 6 Raupen, die ich am 18. Juli zu Lasius niger (ca. 50 Arbeiter, zahlreiche Larven und Puppen) setzte, leben 2 noch heute, 26./1. 1913; die Raupen liegen ruhig, anscheinend ausgewachsen, im Gehäuse jedoch ohne sich zu verpuppen, obwohl sie im warmen Zimmer gehalten wurden. Das stetige allmähliche Verschwinden von Lasius-Larven ließ keinen Zweifel, daß dieselben von den Raupen verzehrt wurden; der Darminhalt in Abessinien konservierter Tiere zeigte ebenfalls Reste von Larvenhäuten, mehr aber kleine Bruchstücke der Chitinbekleidung ausgewachsener Ameisen oder Puppen, ferner oftmals die fast unversehrten Hartteile von Milben, Erd- und Sandpartikel u. dg]. - Diese Raupen dürften sich meist im Abfallnest aufhalten und wohl eher den nur gelegentlich schädlichen, im allgemeinen indifferenten Einmietern zuzuzählen sein als den eigentlichen Synechthren. Nach zahlreichen Funden sind sie fraglos gesetzmäßig myrmecophil.

b) Die Raupen.

Da die Aufzucht der Raupen mir bisher nicht gelungen ist, war es bei der geringen Kenntnis vornehmlich der exotischen Microlepidopteren-Larven und deren Systematik nicht leicht, zu einem 
Urteil über die Zugehörigkeit der Raupen zu kommen. Der Vergleich mit bekannten Formen ließ lediglich den Schluß zu, daß die abessinischen und süd-afrikanischen Chitingehäuseträger wahrscheinlich den Psychiden, die abessinischen Sandgehäuseraupen wohl den Tineiden zuzurechnen seien und letztere vielleicht den Adelinen nahestehen dürften, obwohl sie sich in manchen Punkten höher organisiert zeigen. Bezüglich der süd-afrikanischen Sandgehäuseraupen gewährte das geringe trockene Material keine genaue Untersuchung. Ich gehe auf den Bau der Raupen etwas näher ein, da er manche Eigentümlichkeiten zeigt, welche teils aus der Lebensweise ihre Erklärung finden.

1. Chitingehäuseraupen. Ihre Länge beträgt bis reichlich $7 \mathrm{~mm}$ bei einer größten Breite von $1,7 \mathrm{~mm}$. Der Körper ist im allgemeinen drehrundlich, in der Mitte von oben nach unten etwas

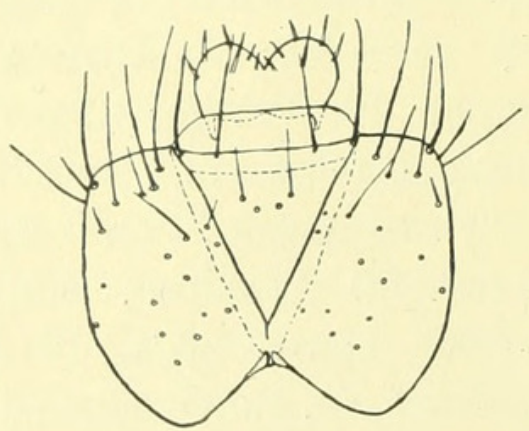

Fig. E.

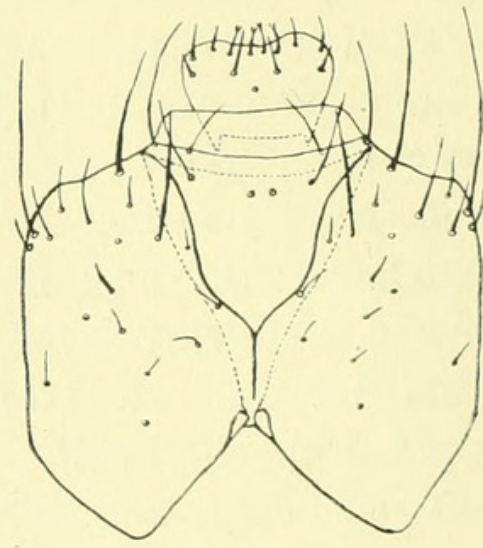

Fig. F.

Fig. E. Kopfkapsel der Chitingehäuseraupe (Abessinien). Beschreibung im Text. Vergr.

Fig. F. Kopfkapsel der Sandgehäuseraupe (Abessinien). Vergr.

zusammengepreßt. Der Kopf und die beiden ersten Thoraxsegmente sind stark chitinisiert, schwarz gefärbt, das 3. Segment ist in der vorderen größeren Hälfte weißlich, weichhäutig und ermöglicht ein rascheres Einziehen der ersten Segmente; die hintere Hälfte ist chitinisiert, schwärzlich. Der ganze übrige Körper ist weiß. - Die Kopfkapsel (Textfig. E) zeigt in ihrem vorderen Teile sehr starke und kräftige, nach hinten zu gar keine ausgebildete Beborstung, jedoch einige Rudimente von solcher nebst Hautsinnesorganen. Das Epistom ist gradseitig, ein gleichschenkliges Dreieck bildend und weit nach hinten reichend. Der Postclypeus trägt die 4 Clypealborsten; das Labrum ist vorn tief eingeschnitten, herzförmig und 
trägt 10 starre fast gerade Dolchborsten auf der Oberseite, 4 kräftige Chitinzähne und zahlreiche feinere zerstreute Zähnchen auf der Unterseite. Die Kopfkapsel besitzt lateral vorn 6 Ocellen, zwischen denen 3 Ocellarborsten stehen. Die Antennen sind 4gliedrig (3gliedrig), höchst auftällig kurz und gedrungen; die Entwicklung ihrer Dolchborsten ist sehr stark und vor allem die Ausbildung der Borste des vorletzten Gliedes sehr kräftig (Textfig. Ha).

Den Antennen unmittelbar benachbart liegen die ausgesprochen handförmigen, כzähnigen Mandibeln. Sie erinnern unwillkürlich an die Kiefer gewisser Ameisen. Nahe der Basis besitzen sie 2 kräftige

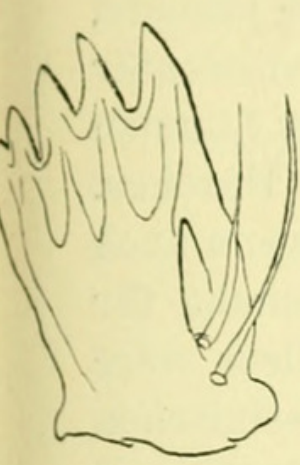

a

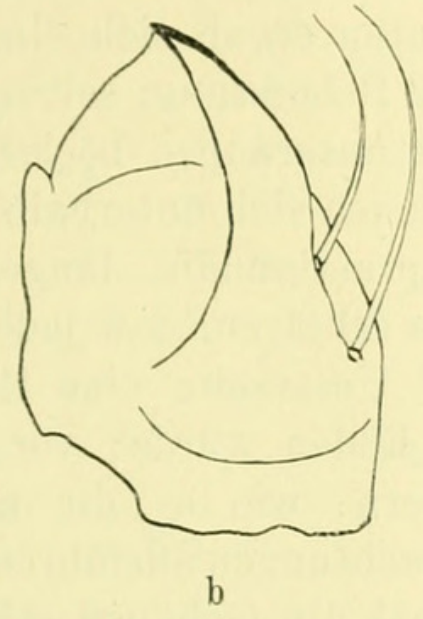

Fig. G.

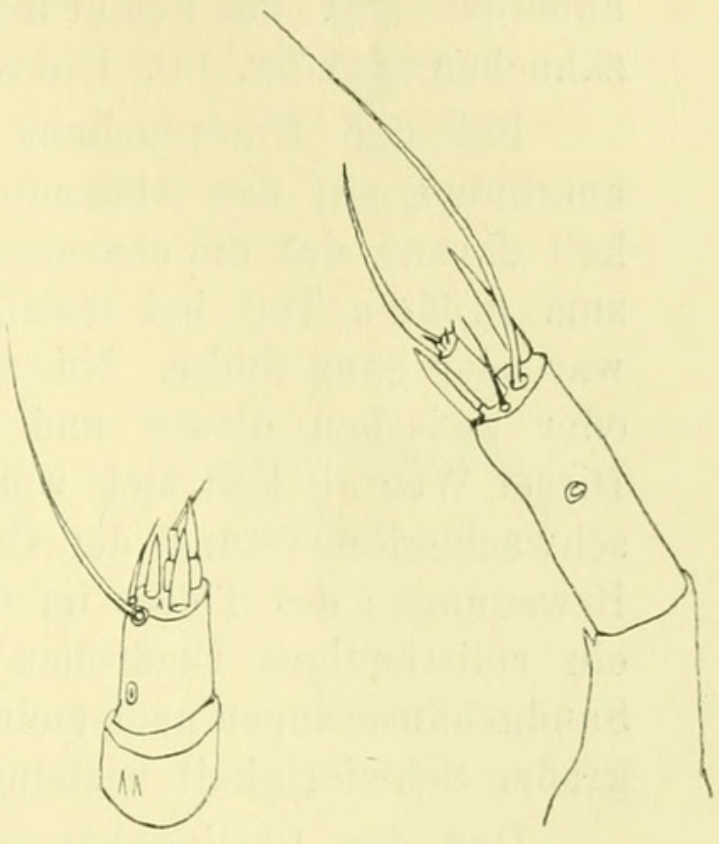

a

Fig. H.

Fig: G. a Mandibel der Chitingehäuseraupe, b der Sandgehäuseraupe (Abessinien). Winkel Obj. 5a, Ok. 2.

Fig. H. a Antenne der Chitin-, b der Sandgehäuseraupe (Abessinien); ebenfalls gleiche Vergr. WInkeL Obj. 5a, Ok. 2.

Borsten (Textfig. G). Die Mandibelform zeigt eine hohe Spezialisierung und feinere Ausarbeitung des ursprünglichen PsychidenCharakters, die eine Erklärung der Kunstfertigkeit des Tieres auf der einen Seite, der räuberischen Lebensweise auf der anderen Seite nahelegt. Das Mentum nebst Spinnkegel und gut ausgebildeten Labialpalpen zeigt keine Besonderheiten. Die Maxillarpalpen haben in der Ausbildung des 3. und 4. Gliedes mehr Ähnlichkeit mit den Tineiden als mit den Psychiden. Die verwachsenen Lobi interni + externi tragen 3 größere und 1 kleinere Dolchborste, das 4. Taster- 
glied ist sehr klein, trägt auf der Spitze ein feines Haar; das 3. Glied besitzt 5 kleine Dolchborsten; sämtliche Glieder sowie die verwachsenen Lobi zeigen je 1 großes Hautsinnesorgan. Stipes und 1. Glied besitzen nach der Innenseite je 1 lange Borste.

Der Kopf ist zum größten Teil in das 1. Thoracalsegment zurückziehbar, eine Eigenschaft, die den Psychiden allgemein zukommt und die eine Erklärung für die mangelnde Beborstung des hinteren Teils der Kopfkapsel gibt. Das Beinpaar des 3. Segments ist das längste, das des 2. etwas, das des 1. viel kürzer und gedrungener. Die Beborstung zeigt keine Besonderheiten, ist aber sehr kräftig; außerdem trägt das Femur innen eine große Zahl stachelartiger Chitinzähnchen (Textfig. Ja). Die Krallen sind scharf, glatt, etwas gebogen.

Bei der Untersuchung der systematisch wichtigen Scleritanordnung auf den Abdominalsegmenten ergab sich eine Schwierigkeit daraus, daß die charakteristische Beborstung, selbst die primäre, zum größten Teil bei sämtlichen Gehäuseraupen höchst rudimentär war oder ganz fehlte. Nur seitlich finden sich unterhalb der Stigmen oder zwischen diesen und den Suprastigmalia längere Härchen. Dieser Wegfall läßt sich wohl daraus erklären, daß jede, wenn auch schwache Beborstung der Ober- und Unterseite eine Hemmung für Bewegungen des Tieres im Gehäuse bilden würde; vor allem wäre ein vollständiges Umdrehen im Innern, wie es die abessinischen Sandgehäuseraupen nach meinen Beobachtungen ausführen können, mit großer Schwierigkeit verbunden, zumal die Gehäuse sehr flach sind.

Daß den Chitingehäuseträgern ebenfalls die Möglichkeit des Sichwendens innerhalb der Wohnung gegeben ist, läßt sich aus dem Vorhandensein der beiden Öffnungen und daraus schließen, daß an beiden Seiten gleichmäßig weitergebaut wird. In den engen Gängen eines Ameisenbaues ist diese Fähigkeit. sich innerhalb des Gehäuses drehen und den Rückweg antreten zu können, ohne das ganze Gehäuse umkehren zu müssen, von großer Bedeutung. -

Die Anordnung der Sclerite ist am klarsten bei Tieren zu erkennen, welche nach vorhergegangener langsamer Härtung in steigendem Alkohol längere Zeit in einer flüssigen Mischung von XylolParaffin belassen, dann kurz mit Xylol abgewaschen und getrocknet wurden.

Das 1. Abdominalsegment zeigt ein großes Dorsalsclerit und jederseits ein Suprastigmale; beim 2. Segment zerfällt das Dorsalsclerit in einen vorderen und hinteren Transversalteil; vom 3. Seg- 
ment an zerfällt das hintere Sclerit in zwei seitliche Teile, eine Anordnung, die bis zum 7. Segment einschließlieh bleibt. Das 8. Segment besitzt gleich dem 2. ein vorderes und ein einheitliches hinteres Sclerit, das 9. und 10. Analsegment nur je ein einheitliches größeres Sclerit. - Die Ventralseite zeigt auf dem 1. Segment vier, dann weiterhin fünf an Umfang wechselnde Ventralsclerite sowie jederseits ein Infrastigmale. Das 3.-6. Segment sowie das letzte besitzen deutliche, wenn auch wenig vorspringende Hakenkranzfüße (Textfig. Ka). Die Zahl der Haken ist zwischen 16 und 22 wech-

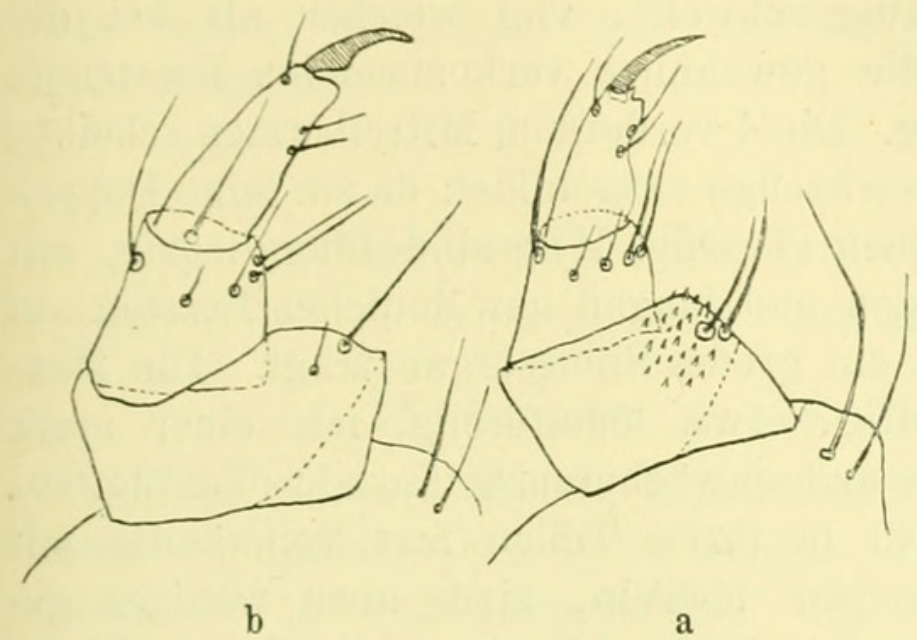

Fig. J.

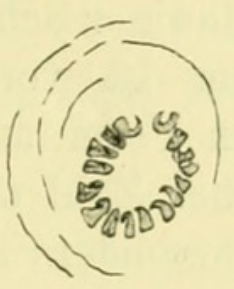

a

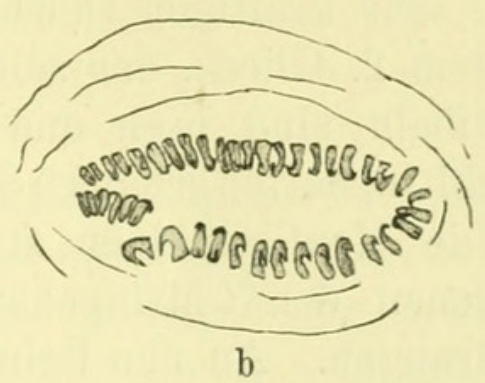

Fig. K.

Fig. J. Linkes Vorderbein: a der Chitingehäuse-, b der Sandgehäuseraupe (Abessinien). WINkEL, Obj. 3a, Ok. 2.

Fig. K. Hakenkranzfüße: a der Chitingehäuse-, b der Sandgehäuseraupe von Port Elizabeth. Winkel, Obj. 3a, Ok. 2.

selnd, Füße des gleichen Segments können einerseits 17, andrerseits 19 oder 20 Haken besitzen. Die Kränze sind meist rundlich kreisförmig.

2. Sandgehäuseraupen (Abessinien). Die größeren Exemplare hatten eine Länge von etwa $1 \mathrm{~cm}$ bei $1,3-1,5 \mathrm{~mm}$ Breite. Im äußeren Aussehen erinnern sie an Adela-Raupen, da die Segmente des Hinterleibes seitlich vorspringen und voneinander durch deutliche Furchen getrennt sind. Die Farbe ist bräunlich-weiß, jedoch kommen auch ganz weiße Stück vor. Die Thoracalseginente sind dorsal schwärzlich, stark chitinisiert, jedoch bleibt an jedem Segment ein vorderer schmaler etwas halbmondförmiger Teil weißlich und weicher; er ist auf dem 1. Segment klein, auf dem 3. reicht er zuweilen über die Hälfte. 
Die Kopfkapsel (Textfig. F) ist wie bei der vorher beschriebenen Form hinten ganz offen. Das Epistom ist hier kürzer, seine Seiten sind geschwungen. und seine Borsten stehen anders zueinander; die sonstige Beborstung des Kopfes ist ähnlich wie bei der Chitingehäuseraupe geschildert wurde; die einzelnen Borsten sind verhältnismäßig schwächer, jedoch greift die Beborstung weiter auf den Hinterkopf über. Mit Sicherheit waren nur 3 Ocellen mit ihren 3 Ocellarborsten aufzufinden; diese geringe Ocellenzahl hängt wohl mit der Lebensweise im Dunkeln zusammen, wie ja auch bei Tineola sogar ein vollständiger Schwund der Ocellen festgestellt werden konnte. Das Labrum ist vorn sanft ausgeschweift, viel weicher als bei der vorigen Form und zeigt die gewöhnlich vorkommenden Borsten in typischer Zahl und Stellung. Die 4 vordersten Mittelborsten scheinen ganz besonders als Sinneswerkzeuge ausgebildet, da sie feine Doppelspitzen haben. Die Antennen (Textfig. $\mathrm{Hb}$ ) sind langgliedrig, mit 2 sehr kräftigen Dolchborsten und langen gewöhnlichen Borsten auf dem 2. Gliede, das seitlich ein großes Sinnesorgan trägt. Die Mandibeln sind breit und kräftig, etwa löffelförmig, mit einer stark chitinisierten scharfen Spitze und schwächerem Seitenzahn (Textfig.Gb). Die Maxillarpalpen stimmen in ihren Teilen fast vollständig mit denen der Chitingehäuseraupen überein, sind aber weniger gedrungen. An den Beinen, welche im Verhältnis zur Größe des Tieres schwächer sind, ist die Beborstung gleichgestellt, aber schwächer entwickelt, die Stacheln des Femurs fehlen, die Krallen sind weniger kräftig.

Von den Scleriten des Abdomens tragen nur einige der Stigmalia sowie die ersten Dorsalia deutliche primäre Börstchen. Alle Abdominalsegmente besitzen bis zum vorletzten dorsal 4 ganze, ziemlich gleichartige Sclerite, d. h. ein Supradorsale anterius, ein Supradorsale posterius sowie rechts und links je ein rundlich ovales Suprastigmale. Vorletztes und letztes Segment besitzen nur je ein Supradorsale. - Auf der Unterseite tragen die ersten beiden Segmente je ein Mittelsclerit; 3.-6. Segment besitzen breitgezogene, sehr wenig vortretende Hakenkranzfüße und nur geringe Scleritspuren. Das 7. Segment trägt zwei kleinere, die folgenden je ein großes Mittelsclerit. Die Kranzfüße besitzen 41-45 Haken, die kaum kenntlichen Nachschieber tragen eine einfache Reihe von 22 bis 28 Haken.

Die süd-afrikanischen Sandgehäuseraupen zeigen, soweit sich erkennen ließ, große Ähnlichkeiten mit den vorgenannten, 
vornehmlich in Bildung der Mundteile. Die Mandibeln sind von gleicher Form, jedoch stumpfer und ohne deutlichen Seitenzahn; ebenso sind Epistom und Labrum ähnlich gestaltet, jedoch sind beiderseits je 6 große Ocellen vorhanden. Die primäre Beborstung ist stärker und deutlich, die Scleritanordnung war nicht mehr kenntlich. Die Gestalt der Kranzfüße und Nachschieber sowie die Zahl der Haken erwies sich als übereinstimmend. Auf alle Fälle ist eine sehr nahe Verwandtschaft zwischen der abessinischen und der südafrikanischen Form vorhanden.

Sämtliche Raupen verpuppen sich, soweit ein Urteil möglich, ohne weitere Gespinstbildung nach einer letzten Häutung innerhalb ihres Gehäuses.

\section{Eine neue myrmecophile Eucharinide.}

(Fam. Chalcididae.)

\section{Psilogaster fraudulentus n. sp.}

Totus cyaneus, antennis nigris, articulo secundo fulgido; femora in medio late cyaneoviridea, apice eorum, tibiarum tarsarumque art. 1-3 fulvis. art. 4 et 5 obscurioribus. Caput, pro- et mesothorax grosse punctata; axillae, scutellum dorsellumque maxime coriacea; metathorax et pedicellium densius subtiliter punctata. Alae translucidae, vitreae, breviter pilosae.

ऽ, 우: $2,7-3 \mathrm{~mm}$.

Dem $P$. cupreus Blanch. nach Fühlerbildung und Körperbau anscheinend nahe verwandt, unterscheidet sich fraudulentus von ersterem durch die geringere Größe und andere Färbung des Körpers der Flügel und Beine.

Die Fühler sind 11gliedrig, scheinbar 10gliedrig, da das 2. Geißelglied sehr klein und mit dem 3. eng verbunden ist. Beim 우 bilden die beiden letzten Fühlerglieder eine Art von Keule. Das 3. Geißelglied ist ziemlich lang und gestreckt, die folgenden werden ganz allmählich etwas kürzer, sind jedoch alle unter sich fast gleich. Das letzte Glied des $\widehat{\jmath}$ fast keulenförmig, an der Spitze gerundet. Die ganze Geißel ist seitlich etwas zusammengedrückt. Fühlerfarbe schwarz, dicht weißlich behaart, das 2. Glied (Pedicellus) etwas grünblau schimmernd. Schenkel in der Mitte breit blaugrün, Beine sonst gelbbraun, letzte Tarsenglieder dunkler. Alle Beine schlank, Schenkel kaum verdickt. Je 5 Tarsenglieder, deren 1. das längste, 
das 2. etwa $3 / 4$ von 1. , 3. und 4 . noch kleiner werdend, das 5 . wenig kürzer wie das 1. - Augen halbkuglig vorspringend; Kopf von vorn dreieckig mit einer sehr flachen Einsenkung vom Clypeus zur Mittelocelle. - Clypeus und Einsenkung fast glatt, kaum punktiert, der übrige Kopf tief eingestochen rauh punktiert. Über dem Scheitel von den Seitenocellen zum Augenrand verläuft eine deutliche feine Furche. Oberlippe 4spitzig, jede Spitze mit starrer Sinnesborste. Mandibeln sichelförmig, unsymmetrisch (Textfig. L). Fühler etwa in der Mitte der Einsenkung erhöht eingelenkt.

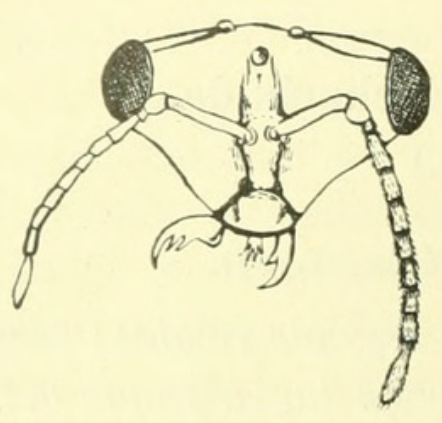

Fig. L.

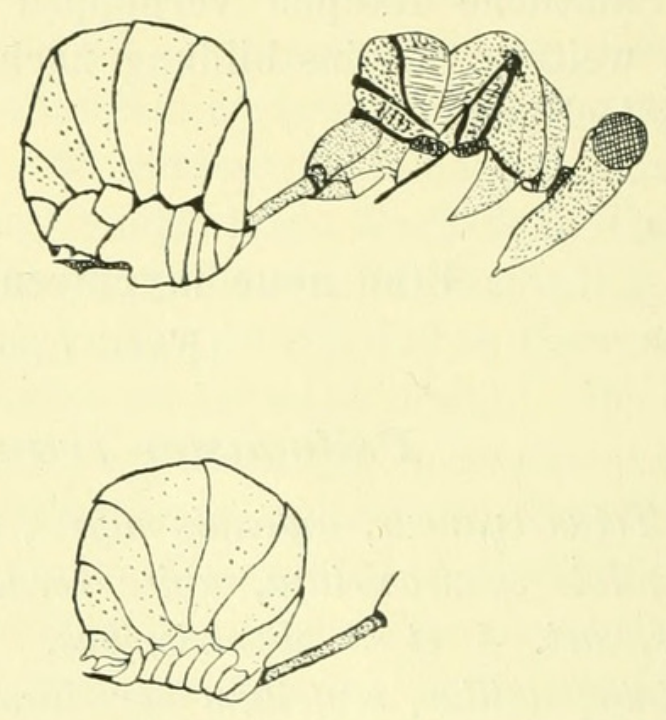

Fig. M.

Fig. L. Kopf von $P$. fraudulentus von vorn. $\sigma^{7}$.

Fig. M. Männchen und Hinterleib des Weibchens von P. fraudulentus, bei gleicher Vergr. Winkel, G., Ok. 2.

Prothorax wenig gewölbt, etwas zusammengedrückt, Mesothorax hochgewölbt, jederseits eine Kuppe bildend, nach hinten steil abfallend und auf dem abfallenden Teil stark längsrunzlig. Scutellum aufgetrieben, sehr tief und grob eingestochen punktiert. Hinterleibsstiel feiner punktiert, lang und dünn, beim + etwas länger als beim $\hat{0}$. Abdomen seitlich stark zusammengedrückt, hochgewölbt, glatt und glänzend, mit sehr zerstreuter spärlicher Punktierung. Larve.

Harrar, Mai, ㅇ, ô; Puppen beider Geschlechter; eine ältere

Mit seinem Wirt, Pheidole megacephala $\mathrm{F}_{\mathrm{AB}}$., steht unser Tier in ebenso engem Zusammenhang wie in Nordamerika die Gattung Orasema Ashmead mit Pheidole kingi André subsp. instabilis Eu. und anderen Pheidole-Arten. Auch die Entwicklung scheint nach 
dem mir vorliegenden Material in ganz ähnlicher Weise zu verlaufen. P. fraudulentus ist ein Brutparasit von Pheidole, aber seine Larven sind nicht, wie man erwarten sollte, Ento- sondern Ectoparasiten. Das einzige mittlere Larvenstadium, das ich erhielt, saß an einer vor der Verpuppung stehenden Pheidole-Larve in der Prosternalregion fest. Die Larve hatte einen eingeschrumpften Körper. Die Psilogaster-Larve wies übereinstimmend mit den Orasema-Larven jederseits auf den Segmenten je 2 Knötchen auf, die den Eindruck von Exsudatknospen machen. Für solche möchte ich nämlich die Knoten in Anspruch nehmen, obwohl mir eine Schnittserie wegen schlechter Konservierung keine Sicherheit über ihren Bau gewähren konnte (vgl. WheELer, 1907, fig. 19, tab. 2). Derartige eigentümliche Knotenbildungen zeigen sich auch an den Puppen von Psilogaster und zwar am stärksten bei den jüngeren Stadien (Taf. 6 Fig. 12). Der Hinterleib ist mit einer Anzahl wallförmiger Erhebungen umgürtet, die seitlich und oben in der Mittellinie Verdickungen aufweisen. Ferner liegen drei kuglige stark vortretende Knötchen zwischen Metathorax und Abdomen über dem Pedicellus; endlich besitzt die Vorderseite des Kopfes oben mehrere kuglige Vorsprünge. An älteren Puppen treten alle Gebilde mit Ausnahme der drei mittleren weniger scharf hervor. Mag man diese Knoten nun vorläufig als eine Art von Exsudatknospen ansprechen oder nicht, sicher ist, daß die Psilogaster-Larven wie die Puppen ihren Wirten höchst angenehm sind; sie werden von ihnen gepflegt und beleckt und wie mir Herr Kristensen ferner schrieb, stürzen sich bei Eröffinung eines Nestes die Pheidole zunächst auf die Puppen und Larven von Psilogaster und suchen sie in die unteren sicheren Nestgänge zu transportieren; erst dann bekümmern sie sich um ihre eigene Brut und suchen diese zu retten. - Der Einfluß des Parasiten auf die Ameisen ist natürlich ein höchst verderblicher. Die von den Psilogaster-Larven befallenen Entwicklungsstadien werden durch Entziehung der Körpersäfte gehemmt und geschwächt, ohne jedoch getötet $\mathrm{zu}$ werden. Sie sind befähigt, sich zwar noch zu verpuppen, bleiben dann aber in der Entwicklung stehen. Eiı Exemplar einer so verkrüppelten Puppe eines Pheidole-Arbeiters lag mir vor. Dieselbe ist ganz zusammengeschrumpft, die Extremitäten, besonders die Fühler, haben ein runzeliges Aussehen, der Thoraxteil ist dünn und schmal, Kopf und Mundteile sind verkümmert. WheELER belegte derartige Wesen mit dem bezeichnenden Namen Phtisergates (bzw. Phtisogyne und Phtisaner). Sie haben keine 
Kraft mehr zur vollen Entwicklung, kommen also nicht über den Puppenstand hinaus; sie liegen unverändert einige Zeit im Nest und werden dann von den Arbeitern zum Abfall gebracht oder aufgezehrt.

Die Übereinstimmung der Lebensweise räumlich so weit voneinander getrennter verschiedener Gattungen wie Orasema und Psilogaster zeigt wiederum deutlich das Bestehen ganz bestimmter Entwicklungsrichtungen. Leider genügen unsere bisherigen Kenntnisse der biologischen Eigentümlichkeiten der interessanten Gruppe der Euchariniden noch in keiner Weise zu einer Schlußfolgerung über die bestimmenden Ursachen dieser Entwicklung; ebenso bedarf es weiterer Beobachtungen und vor allem der Feststellung histologischer Einzelheiten, um zu einer sicheren Erklärung des merkwürdigen einseitigen Freundschaftsverhältnisses der Ameisen zu ihren Parasiten zu gelangen.

\section{Myrmecophila meneliki n. sp. (Gryllodea.)}

Tota brunnea vel fuscobrunnea, pedibus et cercis ochraceis, caput dorsumque setis aureis brevissimis vestitum. Antennae 65-80 articulis compositae, corporis longitudinem aequantes vel paullo superantes. Oculi parvi. Mandibula 4dentata; maxillarum primarum lobus internus dente longo perspicue arcuato biacuminato armatus. Tibiae anteriores et mediae apice infra spina unica, supra seta crassiore instructae; tibiae posteriores spinis septenis magnis, quarum primae inter se aequales.

Während $M$. meneliki sich in allen anderen Kennzeichen als der Gattung Myrmecophila s. st. zugehörig erweist, zeigt sie eines, welches Silvestri's Gattung Myrmecophilina (1912) zukommt, nämlich den Besitz nur eines Dornes am unteren Ende der Vorder- und Mittelschienen, während die übrigen Myrmecophila-Arten dort stets 2 Dornen aufweisen. Die Mitte des Dornes trägt ein Haar. Ein Metasternalanhang, wie er sich bei IMyrmecophilina ochracea vorfindet, fehlt bei unseren Tieren, ebenso die auffallend starke Beborstung des $\widehat{\jmath}$.

Die Farbe ist bei kleineren Exemplaren dunkler, bei größeren meist heller braun. Über die ganze Rückenlänge zieht sich in der Mitte, am Vorderrande des Thorax beginnend, eine feine helle Linie. Das Pronotum ist so lang wie Meso- und Metanotum zusammen; letztere beiden und das 1 . Abdominalsegment sind unter sich gleichlang. Der Legestachel ragt beim Weibchen weit vor; die unteren Lamina sind am Ende krallenförmig, mit schwachgebogener Spitze; die oberen Lamina sind seitlich fein gezähnelt. 
Die Augen sind klein, abgerundet quadratisch, mit 20, in Reihen ziemlich dicht aneinanderliegenden großen Facetten (Textfig. P). In der unteren Außenecke zeigen sich bei stärkerer Vergrößerung durch sehr feine Umrisse leicht angedeutet die Rudimente einer größeren Anzahl von Facetten. Die Hinterbeine (Textfig. N) sind verhältnis-

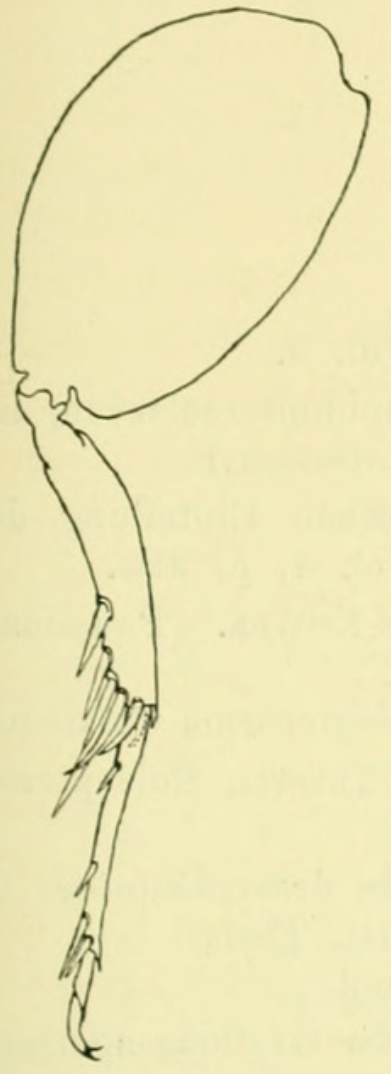

Fig. N.

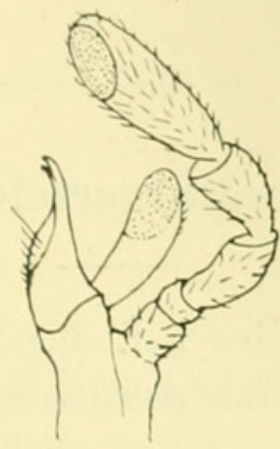

Fig. 0.

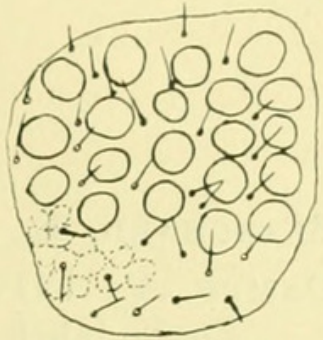

Fig. P.

Fig. N. Linkes Hinterbein von Myrmecoph. meneliki. Winkel, Obj. G., Ok. 4. $36: 1$.

Fig. O. Maxillen und Taster von M. meneliki.

Fig. P. Auge von M. meneliki.

mäßig schlank, die Schenkel knapp halb so lang wie der Körper, die Tibien fast gleichbreit, mit 7 Dornen hinter der ersten Hälfte. Von diesen sind die 3 ersten unter sich gleichlang, ein gutes Kennzeichen der Art, da bei allen bisher bekannten Formen stets der 1. Dorn kleiner als der 2. ist oder nur in einem Falle den 2. an Länge übertrifft. Der 4. Dorn (an der Außenseite stehend) ist weitaus der längste, der folgende erreicht ${ }^{3} / 4$ von dessen Länge, während die letzten etwa den ersten gleichkommen. Das 1. Tarsalglied besitzt einen kleinen Dorn in der Mitte der ersten Hälfte und einen zweiten solchen ein wenig vor der Mitte der zweiten Hälfte; am unteren Ende folgen ein längerer und ein wenig kürzerer Dorn.

Die Länge des 우 beträgt 2,5-4,2 $\mathrm{mm}$ ohne Legescheide, letztere kann bis $1 \mathrm{~mm}$ lang werden; die Breite kann 1-1,6 mm erreichen. ô: $3-3,5 \mathrm{~mm}$ lang.

Abessinien: Harrar, Dire Daoua, Bisa Timo. In zwei Kolonien von Acantholepis capensis canescens 7 우, 1 ô, 2 ältere Larven. Bei Camponotus maculatus F. i. sp. 5 große 우. Bei Pheidole megacephala i.sp. 3 ธิธิ, 1 ㅇ, mehrere Larven. Bei Camponotus rufoglaucus 
Jerd., st. flavomarginatus Mayr 2 우. - Die bei Acantholepis gefundenen Exemplare sind sämtlich, obwohl meist vollständig ausgebildet, kleiner als die aus den Camponotus-Nestern; die PheidoleStücke halten etwa die Mitte zwischen beiden, nur ein $\hat{o}$ war besonders groß.

Bonn, 12. März 1913.

\section{Literaturverzeichnis.}

Blanchard, E., 1840, Histoire nat. des Insectes, Vol. 3.

DAмpF, A., 1910, Zur Kenntnis gebäusetragender Lepidopterenlarven, in:

Zool. Jahrb., Suppl. 12. (Dort auch weitere Literatur.)

ERICHSON, W. F., 1843, Versuch einer systematischen Einteilung der Nitidularien, in: Germar's Ztschr. Entomol., Vol. 4, p. 225.

Gestro, R., 1909, Materiali per lo studio delle Fauna Eritrea. Paussidae, in: Bull. Soc. entomol. Ital., Vol. 41, p. $255 \mathrm{ff}$.

-, 1910, Cupedidae, Paussidae, - Pars 5 aus: Coleopterorum Catalogus.

Lacordaire et Chapuis, 1854, Histoire naturelle des Insects, Coléoptères,

Vol. 2, Paris.

Raffray, A., 1887, Matériaux pour servir à l'étude des coléoptères de la famille des Paussides, in: Arch. Mus. Hist. nat. Peris.

-, 1890, Étude sur les Psélaphides, in: Rev. Entomol.

Schimmer, F., 1909, Beitrag zu einer Monographie der Gryllodeengattung Myrmecophila Latr., in: Z. wiss. Zool., Vol. 93.

—, 1911, Eine neue Myrmecophila-Art aus den vereinigten Staaten, in: D. entomol. Ztschr., 1911.

Schmiedeknecht, Familie Chalcididae, in: Wrtsman, Genera Insectorum. Wasmann, E., 1894, Kritisches Verzeichnis der Myrmekophilen und Termitophilen Arthropoden, Berlin.

-, 1899, Neue Termitophilen und Myrmekophilen aus Indien, in: D. entomol. Ztschr., Hft. 1, p. 161.

—, 1903, Zur näheren Kenntnis des echten Gastverhältnisses (Symphilie) bei den Ameisen- und Termitengästen, in: Biol. Ctrbl., Vol. 23.

—, 1905, Neue Beiträge zur Kenntnis der Paussiden (No. 142), in: Not. Leyden Mus., Vol. 25.

Westwood, 1845, Arcana Entomologica, Vol. 2.

—, 1876, Thesaurus entomologicus oxoniensis, p. $72 \mathrm{ff}$ : Family Paussidae. WHEELER, 1907, The polymorphism of Ants with an account of some singular abnormalities due to parasitism in: Bull. Amer. Mus. nat. Hist., Vol. 23. 


\title{
Erklärung der Abbildungen.
}

\author{
Tafel 5 .
}

Fig. 1. Paussus anxius n. sp. $9: 1$.

Fig. 2. P. modestus n. sp. $9: 1$.

Fig. 3. P. capreolus n. sp. $9: 1$.

Fig. 4. P. kristenseni n. sp. $5: 1$.

Fig. 5. P. globiceps n. sp. 5:1. a Männchen, b Weibchen.

Fig. 6. Hylotorus caroli n. sp. $9: 1$.

Fig. 7. Nitidopecten comes n. g. n. sp. 6:1. ㅇ (Thorax etwas verschoben).

Fig. 8. Sandgehäuse mit Wirtsameise (Abessinien). 4,5:1.

Fig. 9. Chitingehäuse nebst zugehöriger Raupe (Abessinien). 4,5 : 1 .

Tafel 6.

Fig. 1. Exsudatorgan des Kopfes von Hylotorus caroli. a. $O$ äußere Öffnung. $C r$ Cribellum. $D r$ Drüsenzellen. $G$ Gehirn. Winkel Obj. 5a, Ok. 2. Zeichenapparat nach AвBÉ, Objekttischhöhe.

Fig. 2. Mündungsporen des Cribellum von oben, mit Secretresten. $P$ Pore. Winkel Obj. 7a, Ok. 4.

Fig. 3. Einzelne Drüsenzelle aus dem Kopf von Hylotorus, quer. $K$ Kern. Daneben Drüsenbläschen und -kanälchen. Winkel Obj. 7a, Ok. 4.

Fig. 4. Fühlerkeule von Paussus kristenseni in der Aufsicht.

Fig. 5. Dsgl, von P. globiceps.

Fig. 6. Dsgl, von $P$. anxius.

Fig. 7. Dsgl. von P. capreolus. 
218 A. Reichensperger, Zur Kenntnis von Myrmecophilen aus Abessinien.

Fig. 8. Dsgl. von P. modestus nebst Kopf. Fig. 4-8 bei gleicher Vergrößerung.

Fig. 9. Sandgehäuse aus Abessinien in natürlicher Größe; a u. b mit Raupen, c Puppe.

Fig. 10. Sandgehäuse von Port Elizabeth. 1:1.

Fig. 11. Chitingehäuse aus Abessinien mit hervorkommender Raupe. 1:1. Daneben Wirtsameise.

Fig. 12. Jüngere Puppe von Psilogaster fraudulentus. ca. 17:1. 


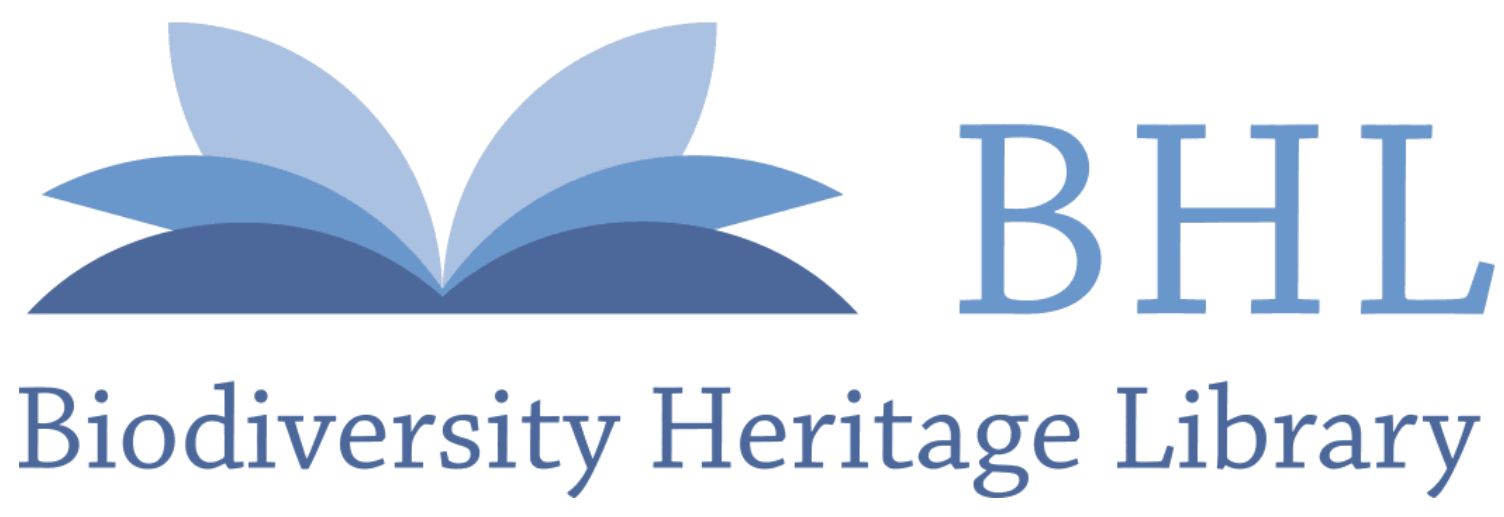

Reichensperger, A. 1913. "Zur Kenntnis von Myrmecophilen aus Abessinien. I." Zoologische Jahrbücher 35, 185-218. https://doi.org/10.5962/bhl.part.16717.

View This Item Online: $\underline{\text { https://www.biodiversitylibrary.org/item/37611 }}$

DOI: https://doi.org/10.5962/bhl.part.16717

Permalink: https://www.biodiversitylibrary.org/partpdf/16717

\section{Holding Institution}

MBLWHOI Library

Sponsored by

MBLWHOI Library

\section{Copyright \& Reuse}

Copyright Status: NOT_IN_COPYRIGHT

This document was created from content at the Biodiversity Heritage Library, the world's largest open access digital library for biodiversity literature and archives. Visit BHL at https://www.biodiversitylibrary.org. 